\title{
Specifics of multi-project management: interaction and resources constraints
}

\author{
Nadezhda Tsvetkova ${ }^{1, *}$, and Iosif Tukkel ${ }^{1}$ \\ ${ }^{1}$ Peter the Great Saint-Petersburg Polytechnic University, Saint-Petersburg, Russia
}

\begin{abstract}
Multi-project management is fundamentally different from the control of a particular project or a set of slightly interconnected projects in terms of complexity and specifics. In multiproject management of the company production it is important to analyze the innovation interaction and its impact on the commercialization stage. A multiparameter factor of innovations interaction was introduced. The optimization problem which considers this factor was mathematically defined. The solution of this problem produces a schedule of innovations launches. This problem definition allows updating the objective function that corresponds to the aims of a manufacturing company. For example, it can help maximize the number of interdependent innovations with restrictions to current tangible and intangible resources or minimize the number of used tangible resources at a fixed number of innovations implemented. In order to verify the optimization problem an evolutionary approach based on genetic algorithm and local search is used. The verification was performed by the Solver a Microsoft Excel add-in. The readiness for practical use of the proposed solution was proved by the experiment.
\end{abstract}

\section{Introduction}

A multi-project management differs fundamentally in problem complexity and specifics from the control of a particular project (mono-project) or a set of slightly interconnected projects both in terms of the rules of resources allocation and possible conflict of local targets.

When constructing a multi-project control system it is important to analyze how the projects interact on each other and how this interaction may have an impact on a product commercialization stage of a manufacturing company production.

A lot of scientific papers of many scientists such as V. Anshin [1], R. Archibald [2], K. Clark [3], R. Cooper [4], S. Edgett [4], A. Kozlovskiy [5], D. Novikov [6], S. Wheelwright [3] et al. were dedicated to the case study of project selection and portfolio construction.

Project interaction issues have been studied by such scientists as M. Dickinson [7], S. Grasman [8], W. Lomax [9], B. Pelegrín [10], D. Pertcev [11], Sh. Srinivasan [8] et al.

It should seem that the above and other researchers have exhausted the problem of determining priority of projects launch in a portfolio. However a specific feature arises in respect to innovations portfolio in the first instance. Innovations interdependence needs extra research related to a specific nature of its interaction.

This paper is devoted to development of such control over sequence of innovations launch that would assume the mentioned specifics as well as limitations of a manufacturing company resources.

\section{Necessary definition}

Let's introduce two descriptors of potential interaction of innovations in a portfolio:

- positive interaction leading to increase in total effect of a portfolio implementation;

- negative interaction leading to decrease in total effect of a portfolio implementation.

There is another definition of the negative impact called cannibalization $[10,12]$.

The required resources for implementation of multiproject control are divided into two types as follows: tangible and intangible [13].

1. The tangible resources $R$ mean a set of conditions enabling to succeed in each project's objective. A distinctive feature of the tangible resources of a project is its time dependence $t$. The basic types of these resources are as follows:

- financial feasibility of a project;

- technological resources;

- human resources;

- information resources.

When analyzing a portfolio of innovations $U$ the tangible resources $R$ are set with two parameters:

- available stock $Q_{i}(t)$ at the moment $t$ of the resource of type $r_{i} \in F$;

- demand $D_{i i}(t)$ at the moment $t$ for the resource of type $r_{i} \in E$ to implement a project $u_{i} \in U$.

*Corresponding author: nadezhdaat@gmail.com 
2. Intangible resources are the ones that initially belong to a project, do not depend on project time $t$ and are not material. Intangible resources include intellectual property assets: essence, innovation idea. Intangible resources separation is required for recording project interaction, its impact on future volume innovative product sales market and on a company competitiveness and so on.

The introduced interaction factor $a_{k, l}$ will be determined as follows.

Innovations portfolio that has been built without consideration of project interaction is defined as $U=\left\{u_{1}, u_{2}, \ldots, u_{n}\right\}$. The groups of dependent projects on intangible resources are found out in a portfolio using an expert approach. A set of these groups is determined as $Z \subseteq U$, where:

- $\quad Z=\left\{Z_{1}, Z_{2}, \ldots, Z_{k}, \ldots, Z_{\bar{l}}, \quad Z_{1} \cap \ldots \cap Z_{\tau}=\emptyset\right.$, $Z_{k}=\left\{Z_{k \uparrow}, \ldots, Z_{k r m}, \ldots, Z_{k s}\right\}, Z_{k} \subseteq Z$;

- $k$ - index number of a group of dependent projects, $k=1,2, \ldots, \zeta$

$-\zeta-$ number of groups of dependent projects, $1 \leq \zeta \leq n / 2$

- $\mathrm{m}-$ index number of a dependent project, $m=1,2, \ldots, \xi$

- number of dependent projects in a group, $1<\varepsilon \leq \gamma$.

A project $Z_{l} \in Z_{k}$ and a subgroup of projects $Z_{k \cdot i}=\left\{Z_{k m} \in Z_{k} \mid Z_{k i} \subseteq Z_{k} \backslash Z_{1}, m \neq I\right]$ will be stand out in each group of dependent projects $Z_{l}$, where $l$ is an index number of a group at issue, $l=1,2, \ldots, \varepsilon$.

Interaction factor $a_{k, l}$ is calculated for each group of dependent projects $Z_{L}$ and demonstrates the extent of impact subgroup of projects $Z_{k r}$ on the project $Z_{l} \in Z_{k}$, $a_{k, i}>0$.

In order to solve the problems of scheduling it is also important to consider for the extent of the impact on a change of a project $z_{1}$ completion date with respect to completion dates of the projects $Z_{t r}: f=f_{b}-f_{i}, f \in \mathbb{Z}$. Considering the above the factor is defined as $a_{k . l .}$.

If $a_{k . f . f}<1$, then a concerned subgroup $Z_{k r}$ has a negative impact on innovation project $Z_{l}$, otherwise, if $a_{k . \text { I.f }}>1$, a subgroup $Z_{r r}$ has a positive impact. No impact at $a_{k . l . f}=1$.

Intangible resources determination results in introducing interaction factor $a_{k, l}=a_{k, l}(G, C, O, M)$ into a project identification summary, where:

- purpose of innovation $G: 1$ - similar, 0,5 - inter crossing, $0-$ different. If $G=[$, interaction is not considered anymore;

- innovation category $C$ : at what stage of a commodity life cycle an innovation is implemented (innovation of end product, processes, procedures, cycles);

- kind of innovation $O$ : value of made variations (modifying, improving, breakthrough, integrating);

- innovation scale $M$ : relation of variation value to a sold product value (global, local).

The stated parameters describe an essence of products of innovation projects and must be considered in a project identification summary. The interaction factor is determined by experts for a certain innovation portfolio.

The factor $a_{k . l .1}$ is a function of many parameters. An expert analysis shall be carried out according to iteration algorithm in this case, for example, [14, 15].

In order to assess an efficiency of multi-project management it is necessary to define an objective function of control.

Let $X_{v}$ be the effect of implementation of independent projects in the portfolio $U$ and $X_{3}$ be the effect of implementation of dependent projects. The objective function will be as follows:

$$
X=X_{V}+X_{3} \rightarrow \max
$$

So the defined objective function allows for providing a content of the effect with different meaning depending on current tasks of a manufacturing company. For example:

- maximization of the number of interdependent innovations with restrictions to current tangible and intangible resources.

- minimization of the number of used tangible resources at a fixed number of innovations implemented.

A problem of determining a sequence of launch of the project in the innovation portfolio comes down to defining a problem of scheduling. In general, a problem of scheduling can be laid down as follows. Using some set of resources some fixed multiparameter system of tasks (works, projects) shall be carried out a purpose of which is to optimize the selected objective function.

\section{Mathematical formulation of a problem}

Let $n$ projects be available in the innovation portfolio $U=\left\{u_{1}, u_{2}, \ldots, u_{n}\right\}$ as before, in which project interaction has not been taken into account yet. All the projects are supposed to be carried out without interruptions.

Let $T=\{1,2, \ldots, \tau\}$ be a set of time units admissible for a schedule, within which limitations may be carried out. Then a duration $\tau \in T$ is a desirable and limit time of implementation of all projects in a portfolio, $\bar{\tau} \in \mathrm{I}$ is a time supposed by the algorithm. Completion time $t_{i} \in T$ of each project $u_{i} \in U$ can be defined in days, weeks, months depending on a project scale.

Each project is described by such parameters as $S_{-}^{-}$ number of a starting event of a project $\boldsymbol{u}_{i}, \boldsymbol{f}_{1}$ - number of final event of a project $u_{i}$, thereat $s_{i}, f_{i} \in T$.

A set of $Z \subseteq U$ is a set of innovation projects determined as dependent according to intangible resources, where $Z=\left\{Z_{1}, Z_{2}, \ldots, Z_{k}, \ldots, Z_{\bar{l}}, \quad Z_{1} \cap \ldots \cap Z_{Z}=\emptyset\right.$, $Z_{k}=\left\{Z_{k+1}, \ldots, Z_{k m,}, \ldots, Z_{k s}, Z_{k} \subseteq Z ; \quad k\right.$ is an index number of a group of dependent projects, $k=1,2, \ldots, \zeta$; $\zeta$ is a number of groups of dependent projects, $1 \leq \zeta \leq n / 2 ; m$ is an index number of a dependent project, $m=1,2, \ldots, \varepsilon ; \varepsilon$ is a number of dependent projects in a group, $1<\varepsilon \leq \gamma$. 
The interaction factor $a_{k . l .1}$ is introduced in the given range of endings $f=f_{k}-f_{\mathrm{i}}$ for each pair consisting of subgroup of projects $Z_{k i}=\left\{Z_{k m} \in Z_{k} \mid Z_{k i} \subseteq Z_{k} \backslash Z_{3}, m \neq l\right]$ and the project $Z_{7} \in Z_{l}$.

Let $R=\left\{r_{1}, r_{2}, \ldots, r_{o}\right\}$ be a set of types of tangible resources required for project implementation and having direct impact on launch sequence in a portfolio. Then $Q_{i}(t)$ is a stock of resource type $r_{i} \in K$ available in a given time, $D_{i j}\left(t^{\prime}\right.$ ) is a demand for the resource $r_{i} \in K$ to implement the project $u_{i}$.

A portfolio $U$ may include independent projects according to intangible resources as well: $Y \subset U$ is a set of independent projects, $Y=\left\{y_{1}, \ldots y_{v}\right\}, 0 \leq v \leq n-2$.

Then a sum of dependent and independent projects will be equal to the number of all projects in a portfolio $n$.

Let $X_{l}^{Y}$ be an effect of implementation of the independent project $y_{i}$ and $X_{l}^{\bar{Z}}$ be an effect of implementation of the dependent project $Z_{1}$.

Vector $S=\left(s_{1}, s_{2}, \ldots, s_{n}\right)$ is called an implementation schedule of the projects in a portfolio. The schedule $S$ is called admissible, if:

- at any specific time $t \in I$ the demand for resources for project implementation will be met for $\forall r_{i} \in K$ $\sum_{i=1}^{n} D_{i j}(t) \leq Q_{i}(t), t \in T$ and limitations assume all types of tangible resources at that;

- the project $u_{i}$ can be started within the period no later than a difference between a number of available periods $\tau$ and project duration $t_{1}$ plus one period, as the counting begins with one: $1 \leq s_{i} \leq \tau-t_{i}+1$.

The effect of independent project implementation is equal to: $X_{Y}=\sum_{i=1}^{\gamma} X_{i}$

The effect of implementation of interconnected projects depends on the project interaction factor: $X^{Z}=\sum_{m=1}^{\zeta} X_{m}^{Z}$, where: $X_{m}^{Z}=\sum_{a=1}^{\varepsilon} a_{k \perp f i}{ }^{*} X_{m r \text {. }}$

As shown above, the objective function (1) is equal to:

$$
X=X_{v}+X_{3} \rightarrow \max
$$

An optimum launch time shall be estimated, that means such $s$ for each project shall be found that will provide for maximum value of the target function $X$ and meet all limitations.

\section{Optimization problem solving}

The defined optimization problem is demonstrated as a diagram (fig. 1).

The defined discrete optimization problem in the context of set resource limitations is NP-hard [16-18]. Due to complexity of the problem it makes sense to use algorithms such as approximation methods [19-21] that base on priority rules, Lagrange relaxations, evolution ideas, local search etc.

Approximation methods are frequently used in a scheduling problem solving [22-27].

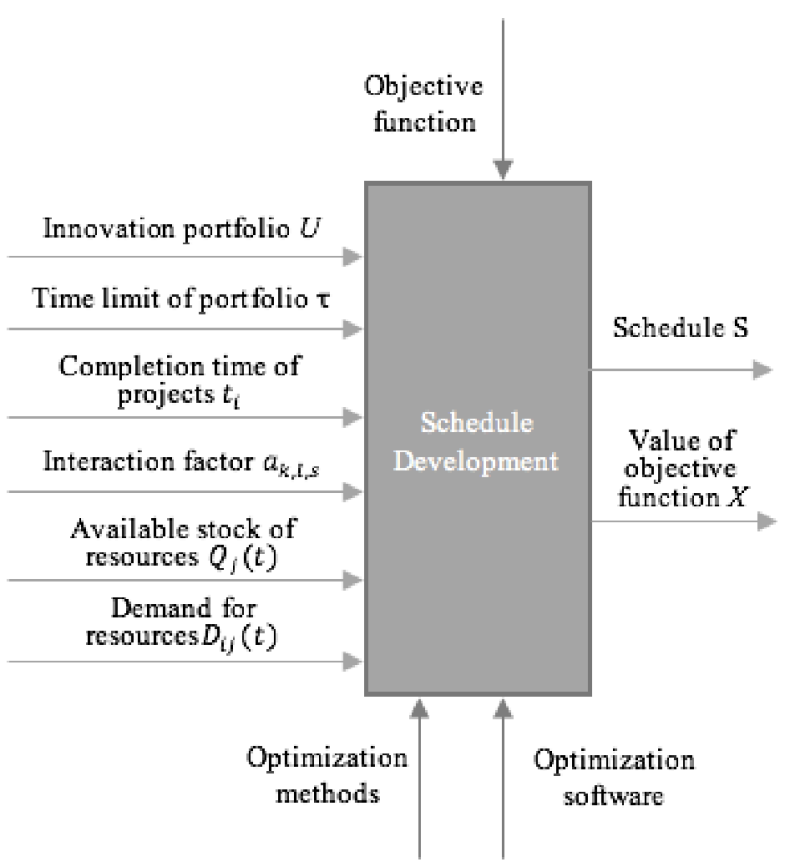

Fig. 1. Problem diagram.

Nowadays there are a number of tools allowing for solving the defined optimized problem in the software market.

For the purposes of the study the widely available Microsoft Excel add-in the Solver was selected for verification of the optimized problem (fig. 2). The evolutionary approach based on genetic algorithm and local search incorporated into the Solver [28] was used. A genetic algorithm is an heuristic algorithm of searching reminding biological evolution. The specific feature of the local search method is that the selection of new decision at each iteration of the process is made not deterministically, but as a result of implementation of some random process $[18,21]$.

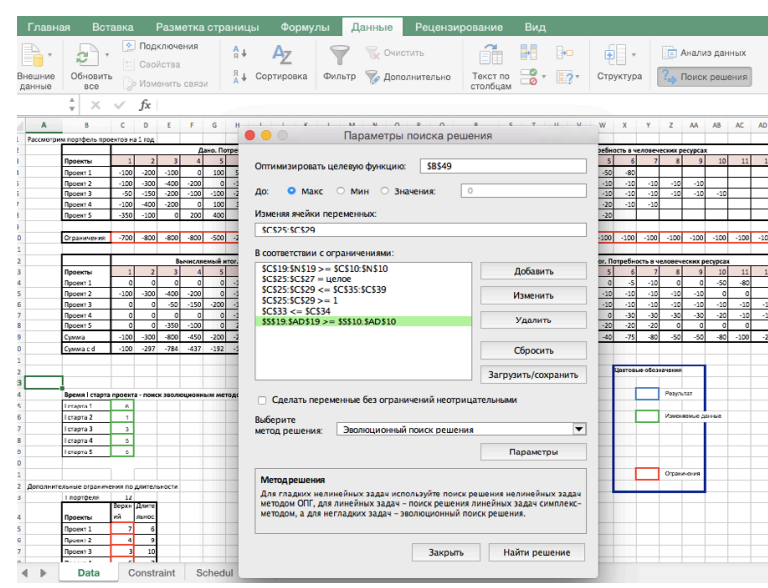

Fig. 1. Examples of problem solving in MS Excel.

The defined optimization problem was verified both on test and real innovation portfolio. Finally the opportunity for improvement of multi-project management efficiency due to taking innovations interaction into account was confirmed. 


\section{Conclusion}

Unlike existing approaches to multi-project control the proposed solution allows for increasing efficiency by virtue of consideration of innovation interaction on intangible resources. A multiparameter factor of innovations interaction was introduced. The optimization problem which considers this factor was defined in terms of mathematics. The solution of this problem produces a schedule of innovations launches. This problem definition allows to update the objective function that corresponds to the aims of a manufacturing company. For example, it can help to maximize the number of interdependent innovations with restrictions to current tangible and intangible resources or minimize the number of used tangible resources at a fixed number of innovations implemented.

In order to verify the optimization problem an evolutionary approach based on genetic algorithm and local search is used. Verification was performed by the Solver a Microsoft Excel add-in. Readiness for practical use of the proposed solution was proved by the experiment.

\section{References}

1. V.M. Anshin, I.V. Demkin, I.M. Nikonov, I.N. Tcarkov, Managing Portfolios of Projects under Uncertainty (MATI, Moscow, 2008)

2. R.D. Archibald, S. Archibald, Leading \& Managing Innovation. What Every Executive Team Must Know about Project, Program \& Portfolio Management (West Conshohocken, Infinity Publishing, 2013)

3. K.B. Clark, S.C. Wheelwright, Managing New Product and Process Development: Text and Cases (Free Press, NY, 1993)

4. R.G. Cooper, S.J. Edgett, E.J. Kleinschmidt, Portfolio management for new products (Basic Books, 2001)

5. A.N. Kozlovskiy, A.O. Nedosekin, Z.I. Abdulaeva, Portfolio management of $R \& D$ projects (SpbSTU, Saint-Petersburg, 2016)

6. D.A. Novikov, I.V. Gontareva, R.M. Nizhegorodcev, Project management (Librokom, Moscow, 2009)

7. M.W. Dickinson, A.C. Thornton, S. Graves, IEEE Transactions on Engineering Management, 48(4), 518-527 (2001)

8. S. Srinivasan, S. Ramakrishnan, S. Grasman, International Journal of Business Innovation and Research, 1, 92-110 (2006)

9. W. Lomax, K. Hammond, R. East, M. Clemete, Journal of Product \& Brand Management, 6(1), 2739 (1997)

10. B. Pelegrín, P. Fernández, M.D.G. Pérez, Annals of Operations Research, 1-19 (2014)

11. D.V. Pertcev, Modern Management: Theory and Practice, 59-69 (2008)
12. J. Heskett, Marketing (Macmillan, New York, NY, 1976)

13. I.L. Tukkel, S.A. Golubev, A.V. Surina, N.A. Tsvetkova, Methods and tools of management of the innovative development of industrial Enterprises (BHV, Saint Petersburg, 2013)

14. G. Rowe, G. Wright, Int'1 Journal of Forecasting, 15, 353-375 (1999)

15. S.N. Jashin, I.L. Tukkel, E.V. Koschelev, Y.S. Korobova, Y.V. Zaharova, Development and decision-making in the management of innovation (N. Novgorod, 2017)

16. J. Blazewicz, J.K. Lenstra, A.H.G. RinnooyKan, Discrete Applied Mathematics, 5, 11-24 (1983)

17. P. Brucker, A. Drexl, R.H. Mohring, K. Neumann, E. Pesch, European Journal of Operational Research, 112(1), 3-41 (1999)

18. Y.A. Zak, Applications of the theory of scheduling and traffic routing (Librokom, Moscow, 2012)

19. C. Artigues, S. Demassey, E. Neron, Resourceconstrained project scheduling: models, algorithms, extensions and applications (Wiley-ISTE, 2007)

20. A.A. Lazarev, E.R. Gafarov, Scheduling Theory: problems and algorithms (MSU, Moscow, 2011)

21. M. Uetz, Algorithms for Deterministic and Stochastic Scheduling (Cuvillier Verlag, 2002)

22. S. Asta, D. Karapetyan, A. Kheiri, E. Özcan, A.J. Parkes, Information Sciences, 373, 476-498 (2016)

23. Ph. Baptiste, C. Le Pape, W. Nuijten, Constraintbased scheduling: applying constraint programming to scheduling problems (Kluwer Academic Publ., 2001)

24. R.K. Chakrabortty, R.A. Sarker, D.L. Essam, IES 2016, 75-86 (2017)

25. R. Kolisch, S. Hartmann, Manuskripte aus den Instituten fu $\ddot{r}$ Betriebswirtschaftslehre (Kiel, Germany, 1998)

26. B. Naderi, Journal of Optimization in Industrial Engineering, 13(13), 65-72 (2013)

27. J. Weglarz, Project scheduling. Recent models, algorithms and applications (Kluwer acad. Publ., Boston, 1999)

28. Basic Solver - Algorithms and Methods Used. http://www.solver.com/content/basic-solveralgorithms-and-methods-used 